\title{
Test of the Law of Gravitation at small Accelerations.
}

\author{
H.Meyer (Bergische Universität Wuppertal), E.Lohrmann, S.Schubert \\ (Universität Hamburg), W.Bartel, A.Glazov, B.Löhr, C.Niebuhr, E. \\ Wünsch (DESY), L.Jönsson (University of Lund), G. Kempf \\ (Hamburgische Schiffbau-Versuchsanstalt).
}

\begin{abstract}
Newton's Law of Gravitation has been tested at small values $a$ of the acceleration, down to $a \approx 10^{-10} \mathrm{~m} \mathrm{~s}^{-2}$, the approximate value of MOND's constant $a_{0}$. No deviations were found.
\end{abstract}

\section{Introduction}

The nature of Dark Matter is one of the central questions in astrophysics at present. Introduced originally to explain the dynamics of galaxies, Dark Matter has found an established place in the Cosmological Model. Still, many questions and difficulties remain, see e.g. [1]. In this context, also alternative explanations are discussed, one of them being MOND (modified-Newtoniandynamics) [2]. MOND assumes, that the gravitational law is modified for small values of the acceleration in the following way:

$$
a_{N}=a \cdot \mu\left(a / a_{0}\right)
$$

Here, $a$ is the acceleration according to MOND, $a_{N}$ is the Newtonian acceleration $a_{N}=G \cdot \mathrm{m} / \mathrm{r}^{2}$, and $a_{0}=1.2 \cdot 10^{-10} \mathrm{~m} \mathrm{~s}^{-2}$ is assumed to be a universal constant [1], 3]. The interpolation function $\mu\left(a / a_{0}\right)$ is $\mu \rightarrow 1$ for $a>>a_{0}$, recovering Newton's Law, and $\mu \rightarrow a / a_{0}$ for $a<<a_{0}$. Apart from these 
asymptotic values the interpolation function is not determined by the theory, but has to be constrained by data.

A relativistic formulation incorporating the MOND theory has been developed by Bekenstein [4].

MOND has so far passed many astronomical tests [1] [3]. Apart from modifying Newton's Law, MOND could also be interpreted as a violation of Newton's second axiom $F=m \cdot a$ [5], irrespective of the nature of the force $F$. This latter aspect has been experimentally checked, using electromagnetic restoring forces, and Newton's axiom verified down to accelerations of $3 \cdot 10^{-11} \mathrm{~ms}^{-2}$ [6] and $5 \cdot 10^{-14} \mathrm{~m} \mathrm{~s}^{-2}$ [7]. Therefore, a possible modification according to MOND must rest with the gravitational force alone. This experiment is designed to test Newton's Law at accelerations of the order $a_{0}$, using only gravitational forces. It has been argued, that such a test is not meaningful in the strong gravitational field of the earth, but, due to a lack of a deeper understanding of MOND, this view is not shared by everybody (see e.g. [3]).

\section{Experimental Procedure}

A schematic view of the experiment is shown in Fig. 1.

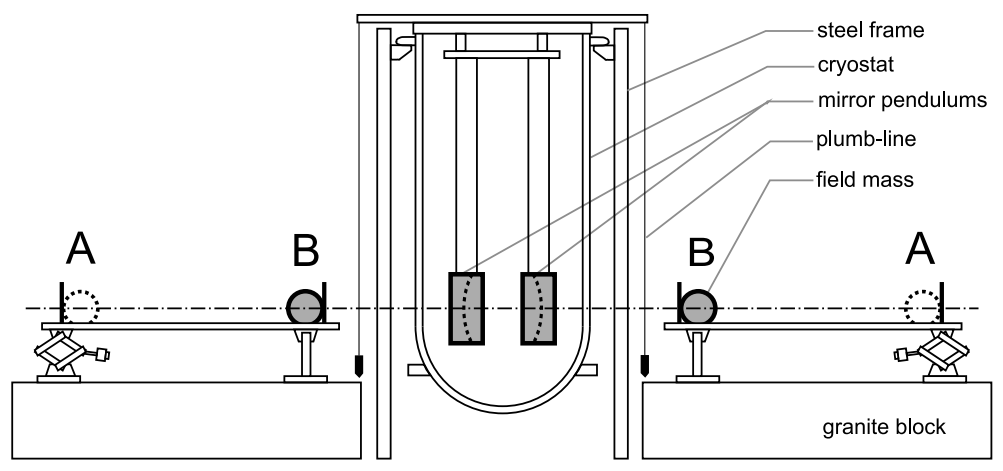

Figure 1: Schematic view of the experiment

The central part of the experiment is a microwave resonator, tuned to a frequency of about $21.3905 \mathrm{GHz}$. The resonator consists of two mirrors with 


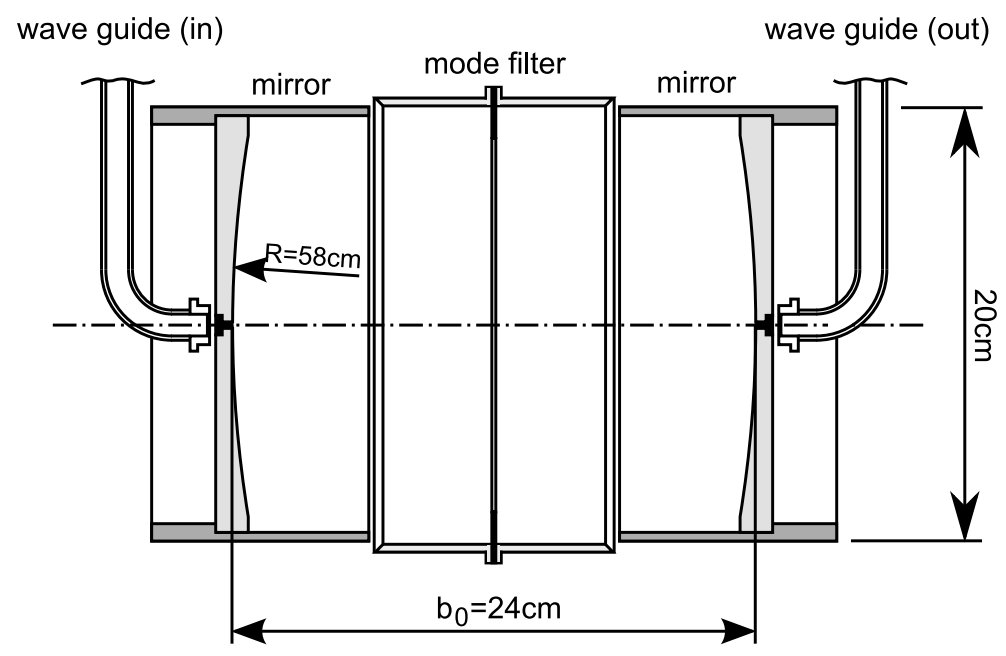

Figure 2: Schematic view of the resonator

spherical surfaces, suspended by tungsten wires of about $3 \mathrm{~m}$ length, resulting in a pendulum period of $3.289 \pm 0.010 \mathrm{~s}$. This part of the detector sits in an evacuated vessel. Two field-masses are positioned outside the vacuum vessel on either side of the resonator, and are periodically and simultaneously moved between a far (A) and a near (B) position. Their gravitational pull results in a small change of the position of the two mirrors, which is measured from the change of the resonance frequency.

A detailed view of the resonator is presented in Fig. 2, showing also the microwave guides.

The apparatus had been built and operated at Wuppertal University for a precision measurement of the gravitational constant [8], [9], [10], [11], [12]. It was later tranferred to DESY and reinstalled with some improvements for the stability of the support [13, [14].

Measurements were carried out with three pairs of field-masses, consisting of spheres of brass, marble and plastic, with masses of $9.02 \mathrm{~kg}, 2.92 \mathrm{~kg}$ and $1.00 \mathrm{~kg}$, respectively. All spheres have the same diameter of $12.7 \mathrm{~cm}$. They were placed at the near position with their centers at $76.6 \mathrm{~cm}$ on the left side and of $77.9 \mathrm{~cm}$ on the right side from the center of gravity of the 
nearest mirror, respectively. The acceleration of a mirror caused by the closer field-mass at the near position was $10.2 \cdot 10^{-10} \mathrm{~m} \mathrm{~s}^{-2}, 3.3 \cdot 10^{-10} \mathrm{~m} \mathrm{~s}^{-2}$, and $1.1 \cdot 10^{-10} \mathrm{~m} \mathrm{~s}^{-2}$ for the three masses, resulting in a change of the distance between the two mirrors from about $0.210 \mathrm{~nm}$ to $0.023 \mathrm{~nm}$. If Newton's Law is correct, the deflections due to the three field-masses must be precisely proportional to their mass values.

Measurements were carried out by moving the left (right) field-masses from the near position at $76.6 \mathrm{~cm}(77.9 \mathrm{~cm})$ to a far position at $213 \mathrm{~cm}(220$ $\mathrm{cm}$ ) every $40 \mathrm{~min}$.

Measurements of the resonance frequency $f_{R}$ were performed every $2 \mathrm{sec}$ by tuning the frequency of the generator to five values around the resonance frequency and recording the resulting amplitude at the exit of the resonator. The resonance frequency was then determined by fitting a resonance curve of the form Equ.(2) to the five amplitude values $U(f)$.

$$
U(f)=U_{\max } \cdot \frac{1}{1+4\left(\left(f-f_{R}\right) / f_{w}\right)^{2}} .
$$

Here $\mathrm{U}(\mathrm{f})$ is the amplitude at frequency $\mathrm{f}, f_{R}$ is the fitted resonance frequency and $f_{w}$ is the resonance width.

The frequency measurements were then averaged over typically 1-2 min. The temperature at the apparatus was kept constant to about 0.1 degree; still the data show a strong drift with temperature, which must be corrected for.

Figure 3 shows an example of a measurement with the $9.02 \mathrm{~kg}$ fieldmasses before and after subtraction of a slow frequency drift. From the frequency measurements a large constant frequency (about $21 \mathrm{GHz}$ ) has been subtracted.

Additional distortions come also from sources like ground movements, occasional earthquakes and waves from the North Sea: Fig. 4 shows as an example the rms noise of single frequency measurements plotted against the significant wave height at the mouth of the Elbe river. 

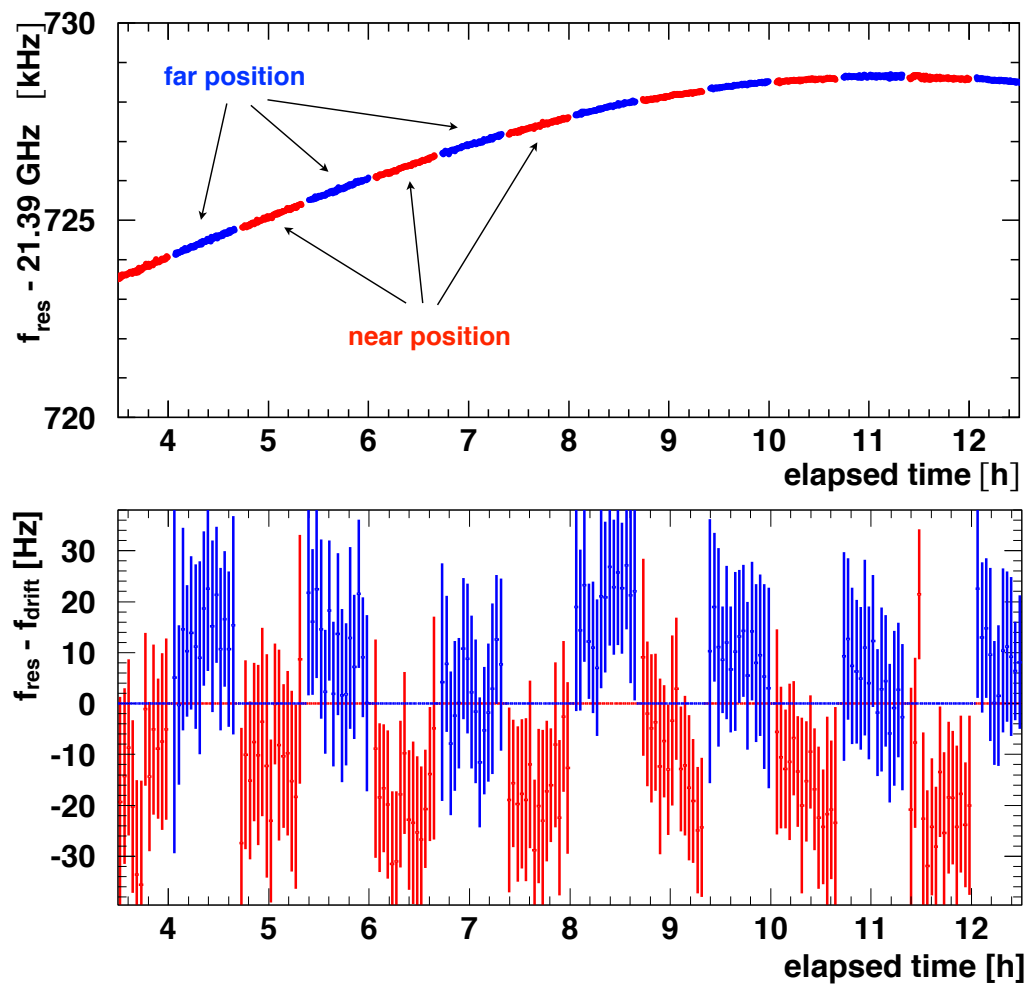

Figure 3: Frequency as a function of time for a measurement with the $9.02 \mathrm{~kg}$ sphere, without (above) and after (below) a background drift subtraction. A large constant frequency offset has been subtracted. 


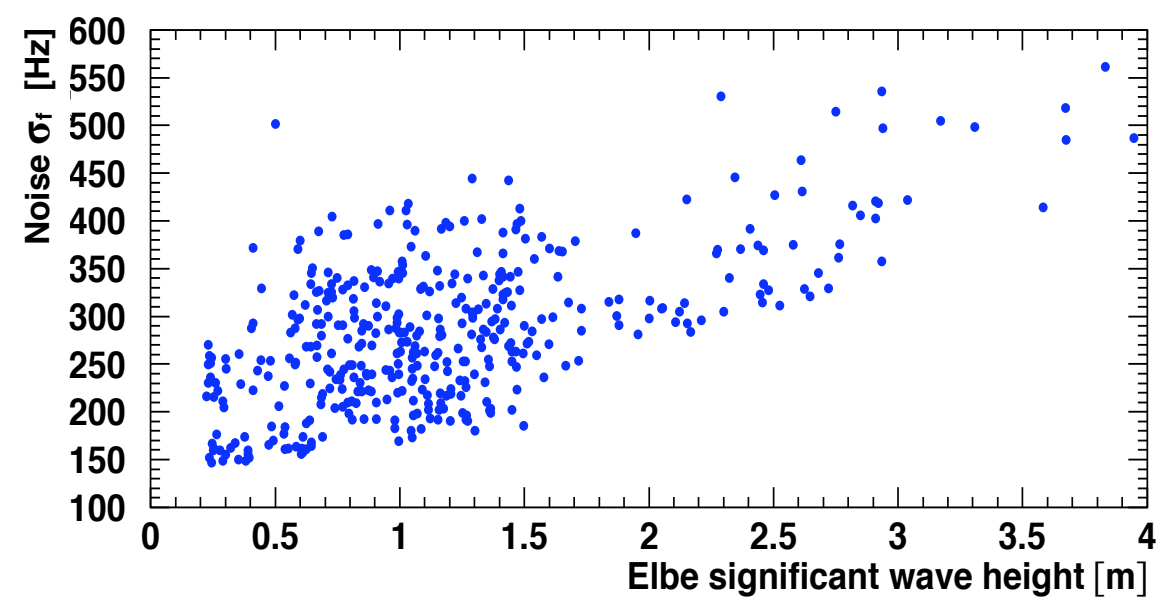

Figure 4: The noise (rms) of a single frequency measurement as a function of the significant wave height at the mouth of the Elbe river.

\section{Results}

The data were evaluated using six different methods of background subtraction, to deal with slow drifts of the resonance frequency and with short-term background variations: Overall polynomial fits (A1, A3), piecewise 3rd order polynomial fit (A5), piecewise 5th order polynomial fit (A6), and sliding average (A2, A4) [14]. For each mass the uncertainties of the mean values in Table 1 were determined from the variance of the results of about 25 independent data runs with an average duration of 12 hours. The differences between the results of different methods for the $9.02 \mathrm{~kg}$ data are somewhat larger than expected from the individual errors and reflect differences in dealing with short-term variations of the background. To deal with it, the uncertainty of the $9.02 \mathrm{~kg}$ data is in the following Fig. 5 and in the calculation of $G$ increased by a factor of 1.5, according to the PDG prescription [15]. All six methods were checked with several sets of Monte Carlo data, to which regular and irregular noise similar to the one observed in the data had been added. All methods were able to reproduce the correct input signal within the uncertainties [14].

Method A1 was used as the central value and the other methods as con- 
sistency checks.

Table 1: Mean frequency shift $\Delta f$ in $\mathrm{Hz}$ for the three field-masses

\begin{tabular}{|l|l|l|l|}
\hline Method & $\Delta f 1.0 \mathrm{~kg}$ & $\Delta f 2.92 \mathrm{~kg}$ & $\Delta f 9.02 \mathrm{~kg}$ \\
\hline A1 & $2.06 \pm 0.25$ & $6.09 \pm 0.24$ & $18.65 \pm 0.40$ \\
A2 & $2.00 \pm 0.44$ & $6.25 \pm 0.32$ & $19.12 \pm 0.54$ \\
A3 & $2.06 \pm 0.38$ & $6.19 \pm 0.34$ & $18.53 \pm 0.60$ \\
A4 & $2.94 \pm 0.48$ & $6.36 \pm 0.31$ & $19.30 \pm 0.61$ \\
A5 & $1.72 \pm 0.59$ & $6.31 \pm 0.36$ & $20.09 \pm 0.51$ \\
A6 & $1.60 \pm 0.25$ & $5.63 \pm 0.23$ & $17.00 \pm 0.56$ \\
\hline
\end{tabular}

Assuming, that the potential effect due to MOND is negligeable for the $9.02 \mathrm{~kg}$ field-masses, the gravitational constant $\mathrm{G}$ can be computed from the frequency shift $\Delta f$ between the far and near positions of the $9.02 \mathrm{~kg}$ fieldmasses. The values of $\Delta f$ given in Table 1 are the sum of the frequency shifts of the right and left field-masses.

The frequency shift $\delta f$ due to one field-mass is given by

$$
\frac{\delta f}{f}=\frac{G M T_{0}^{2} \cdot \Delta\left(1 / r^{2}\right)}{4 \pi^{2} b}
$$

with

$$
\Delta\left(1 / r^{2}\right)=\left(1 / r_{n}^{2}-1 /\left(r_{n}+b\right)^{2}\right)-\left(1 / r_{f}^{2}-1 /\left(r_{f}+b\right)^{2}\right)
$$

Here $f$ is the frequency, $M$ the field-mass, $T_{0}$ the pendulum period of the cavity, $b$ the distance between the two cavities, $r_{n}$ and $r_{f}$ are the distances of the near and far position of the field-mass from the nearest mirror, respectively.

Using method A1 as a reference value, and after a correction taking account of the detailed shape of the mirrors [14, one obtains a value for $G$

$$
G=(6.57 \pm 0.21 \pm 0.11) \cdot 10^{-11} \mathrm{~m}^{3} / \mathrm{kg} \mathrm{s}^{2}
$$

where the first uncertainty is due to the uncertainty of the frequency shift. The second uncertainty is systematic, with the list of systematic uncertainties as given in the table below. The first three entries in the table follow directly from the corresponding measurement uncertainties, the fourth entry 
follows from the estimated accuracy of the integration over the mirrors, and the uncertainty of $b$ was determined from an analysis of the mode spectrum of the resonator.

Table 2: Systematic uncertainties contributing to the measurement of $G$.

\begin{tabular}{|l|l|}
\hline Source & uncertainty in $\%$ \\
\hline pendulum frequency $T_{0}^{-1}$ & 0.66 \\
position of the field-masses $r_{n}, r_{f}$ & 1.48 \\
value of the field-masses $M$ & 0.11 \\
integration over the mass distribution of the mirrors & 0.20 \\
distance between mirrors $b$ & 0.01 \\
\hline
\end{tabular}

This value of $G$ agrees with the world average [15] of $G=6.67428(67)$. $10^{-11} \mathrm{~m}^{3} / \mathrm{kg} \mathrm{s}^{2}$ within the uncertainties.

Predictions from MOND are not unambiguous. We assume, that the forces from each field-mass on each cavity, as calculated from the MOND formula, can be added linearly. With this assumption one can calculate the frequency shifts for the different field-masses and for the different interpolation functions $\mu(x)$, with $a=$ acceleration due to MOND, $a_{N}=$ Newton's acceleration, $x=a / a_{0}, y=\sqrt{\left(a_{N} / a_{0}\right)}$ :

$$
\begin{aligned}
\text { MOND1 [5] } & \mu(x)=x / \sqrt{1+x^{2}} \\
\text { MOND2 [16] } & \mu(x)=x /(1+x) \\
\text { MOND3 [17] } & \mu(x)=6 x / \pi^{2} \cdot \int_{0}^{\pi^{2}} / 6 x z /\left(e^{z}-1\right) d z \\
\text { MOND4 [18] } & a=a_{0} y \cdot\left(1-y^{4}\right) /\left(1-y^{3}\right) \\
\text { MOND5 [4] } & \mu(x)=(\sqrt{1+4 x}-1) /(\sqrt{1+4 x}+1)
\end{aligned}
$$

Fig. 5 shows a comparison of the measurements with the predictions of the five interpolation functions. The normalized frequency shift $\Delta f / M$ (where $M$ is the mass of the field-mass) is plotted for the three field-masses. The error bars on the data points represent the uncertainty of the measured frequency shifts. The width of the bands for the interpolating functions shows the effect of the systematic uncertainties. If Newton's Law is valid, $\Delta f / M$ must be the same for all field-masses. Version MOND5 due to Bekenstein's relativistic 
theory is clearly ruled out (also ruled out by astrophysical observations [16]). The versions MOND1 and MOND2 are only slightly disfavoured.

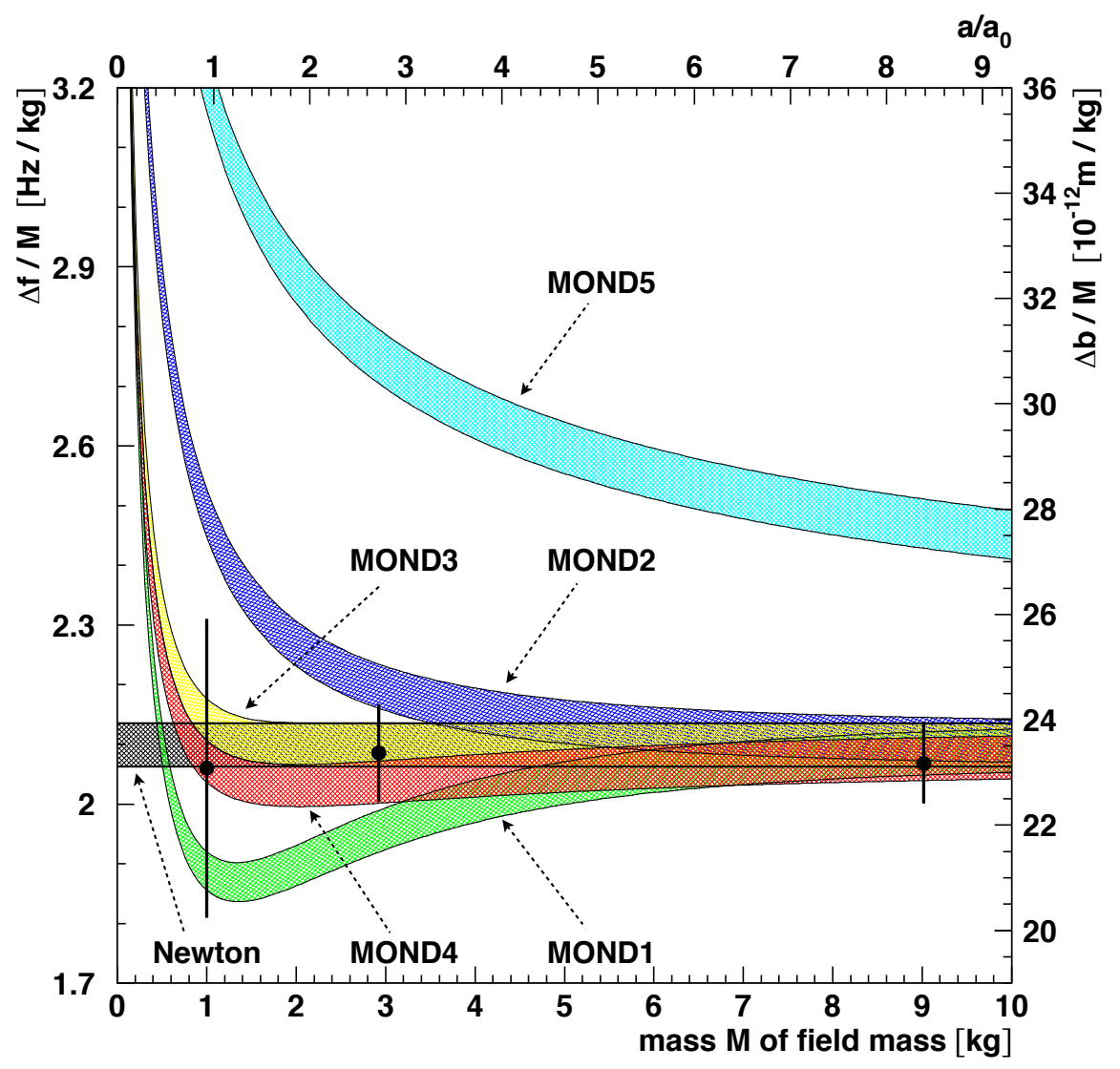

Figure 5: Comparison of different versions of the MOND interpolation function with the measurements. The values of the field-masses $M$ are plotted against $\Delta f / M$, where $\Delta f$ is the frequency shift. The upper horizontal axis indicates the value of the acceleration of one of the mirrors caused by the closer field-mass in the near position in units of the MOND acceleration $a_{0}$. The vertical axis on the right hand side shows the corresponding relative change of the distance between the mirrors. 


\section{Conclusions.}

Newton's Law of Gravitation has been tested for small values of the acceleration, using a pair of pendulums to measure the gravitational attraction. The data cannot refute the MOND theory, but they have successfully probed

Newton's Law down to the MOND acceleration $a_{0}=1.2 \cdot 10^{-10} \mathrm{~m} / \mathrm{s}^{2}$. The accuracy of the measurements will be improved by moving the experiment to an underground location and by a better mechanical support.

\section{Acknowledgments}

It is a pleasure to thank the DESY Directorate for their support. We also acknowledge the assistance of U.Cornett, M.Gil, D.Habercorn, T.Külper, C.Muhl, J.Schaffran, H.J.Seidel and V.Sturm in setting up the experiment, and we thank the technical groups of DESY for their help and advice in many technical questions.

\section{References}

[1] P.Kroupa et al.,arXiv:1006.1647v3; Astronomy and Astrophysics A32(2010) 523

[2] M.Milgrom, Astrophys.J.270(1983)365

[3] R.Scarpa, arXiv:astro-ph/0601478; Conf.Rep.'First Crisis in Cosmology' AIP publ.Vol.822

[4] J.Bekenstein, Phys.Rev. D70(2004)083509, Erratum Phys.Rev. D71(2005)069901

[5] M.Milgrom, Astrophys.J.270(1983)371

[6] A.Abramovici and Z.Vager, Phys.Rev. D34(1986)3240

[7] J.H.Gundlach et al., Phys.Rev.Lett. 98(2007)150801

[8] U.Kleinevoss, H.Meyer, A.Schumacher and S.Hartmann Meas.Sci.Technol. 10(1999)439 ; U.Kleinevoss, H.Meyer, H.Piel and S.Hartmann, Conf. on Precision Electromagnetic Meas. 2002, Conf.Digest 2002 (2002)148 
[9] Ulf Kleinevoss, Dissertation Wuppertal 2002

[10] Harald Walesch, Dissertation Wuppertal 1995

[11] Juergen Schurr, Dissertation Wuppertal 1992

[12] A.Schumacher, Dissertation Wuppertal 1999

[13] Sven Schubert, Diplomarbeit Hamburg 2008.

[14] Sven Schubert, Dissertation Hamburg 2011.

[15] Particle Data Group J.Phys.G37(2010)075021; for the latest value of G see http://pdg.lbl.gov/2011/reviews/rpp2011-revphys-constants.pdf

[16] B.Famaey and J.Binney, arXiv:astro-ph/0506723

[17] X.Li and Z.Chang, arXiv:hep-th/1005.1169v1

[18] S.Mendoza et al., arXiv:astro-ph/1006.5037.v2 